\title{
First report of Leishmania (Viannia) lindenbergi causing tegumentary leishmaniasis in the Brazilian western Amazon region
}

Lilian Motta Cantanhêde ${ }^{1}$, Cristiane Batista Mattos ${ }^{1}$, Camila de Souza Ronconi ${ }^{1}$, Camila Patrício Braga Filgueira ${ }^{2}$, Cipriano Ferreira da Silva Júnior ${ }^{3}$, Claudino Limeira ${ }^{1}$, Helen Paula de Jesus Silva ${ }^{1}$, Gabriel Eduardo Melim Ferreira ${ }^{1}$, Renato Porrozzi ${ }^{2}$, Ricardo de Godoi Mattos Ferreira ${ }^{1}$, and Elisa Cupolillo ${ }^{2}$,*

${ }^{1}$ Fundação Oswaldo Cruz, Unidade Rondônia, Porto Velho, Rondônia, Brazil

${ }^{2}$ Fundação Oswaldo Cruz, Instituto Oswaldo Cruz, IOC, Rio de Janeiro, Brazil

${ }^{3}$ Secretaria de Saúde de Rondônia, SESAU, Porto Velho, Rondônia, Brazil

Received 5 February 2019, Accepted 23 April 2019, Published online 23 May 2019

\begin{abstract}
Tegumentary Leishmaniasis (TL) in the Brazilian Amazon region is associated with several Leishmania species. In this report, we describe two cases of TL related to Leishmania lindenbergi occurring in different locations of Rondônia state. After clinical diagnosis, lesion samples were collected for parasitological diagnoses via direct microscopic visualization, parasite isolation, and PCR. PCR reactions were positive in both clinical samples. Parasite isolation was possible for both patients, and isolates were submitted to species identification by isoenzyme electrophoresis and DNA sequencing. This report is the first to describe human infections caused by L. lindenbergi since the initial description and record of human infection by this species in 2002 .
\end{abstract}

Key words: Leishmania (Viannia) lindenbergi, Tegumentary Leishmaniasis, Western Amazon.

\begin{abstract}
Résumé - Premier signalement de Leishmania (Viannia) lindenbergi causant une leishmaniose tégumentaire dans la région amazonienne de l'ouest du Brésil. La leishmaniose tégumentaire (TL) en Amazonie brésilienne est associée à plusieurs espèces de Leishmania. Dans ce rapport, nous décrivons deux cas de TL liés à Leishmania lindenbergi survenus à différents endroits de l'état de Rondônia. Après le diagnostic clinique, des échantillons de lésion ont été recueillis à des fins de diagnostic parasitologique par visualisation au microscope direct, isolement du parasite et PCR. Les réactions de PCR étaient positives dans les deux échantillons cliniques. L'isolement des parasites a été possible pour les deux patients et les isolats ont été soumis à l'identification de l'espèce par électrophorèse des isoenzymes et séquençage de l'ADN. Ce rapport est le premier à décrire les infections humaines causées par L. lindenbergi depuis la description initiale et le signalement de cas d'infection humaine par cette espèce en 2002.
\end{abstract}

\section{Introduction}

Various Leishmania species act as etiological agents in Tegumentary Leishmaniasis (TL). More than 20 species of Leishmania are known to be infectious to humans. Some of these species are widespread around the world with major concentrations in tropical and subtropical regions. In Brazil, seven Leishmania species have been identified as human pathogens causing TL: one species of the subgenus Leishmania (Leishmania): L amazonensis and six species of the subgenus Leishmania (Viannia): L. braziliensis, L. guyanensis, L. naiffi, L. lainsoni, L. shawi, and L. lindenbergi. All of these species are endemic to the Brazilian Amazon region [9, 13, 14].

The rarest species registered in Brazil is L. lindenbergi, which was reported to cause human infections in soldiers

\footnotetext{
*Corresponding author: elisa. cupolillo@gmail.com
}

performing activities in secondary forests and in a woman from the same area in Belém, state of Pará, Brazil [13]. This single report refers to human infections, but little is known concerning the parasite's biology, including hosts, reservoirs, and vectors. The most likely associated vector is Nyssomyia antunesi due to the abundance of this vector in the area where the soldiers were infected by L. lindenbergi. The description of the clinical features, as well as the course of the infections caused by L. lindenbergi, are also limited, although all infections that were reported presented with localized cutaneous manifestations [13].

\section{Materials and methods}

During the period from 2013 to 2017, more than 500 patients with suspected Cutaneous Leishmaniasis were seen at 


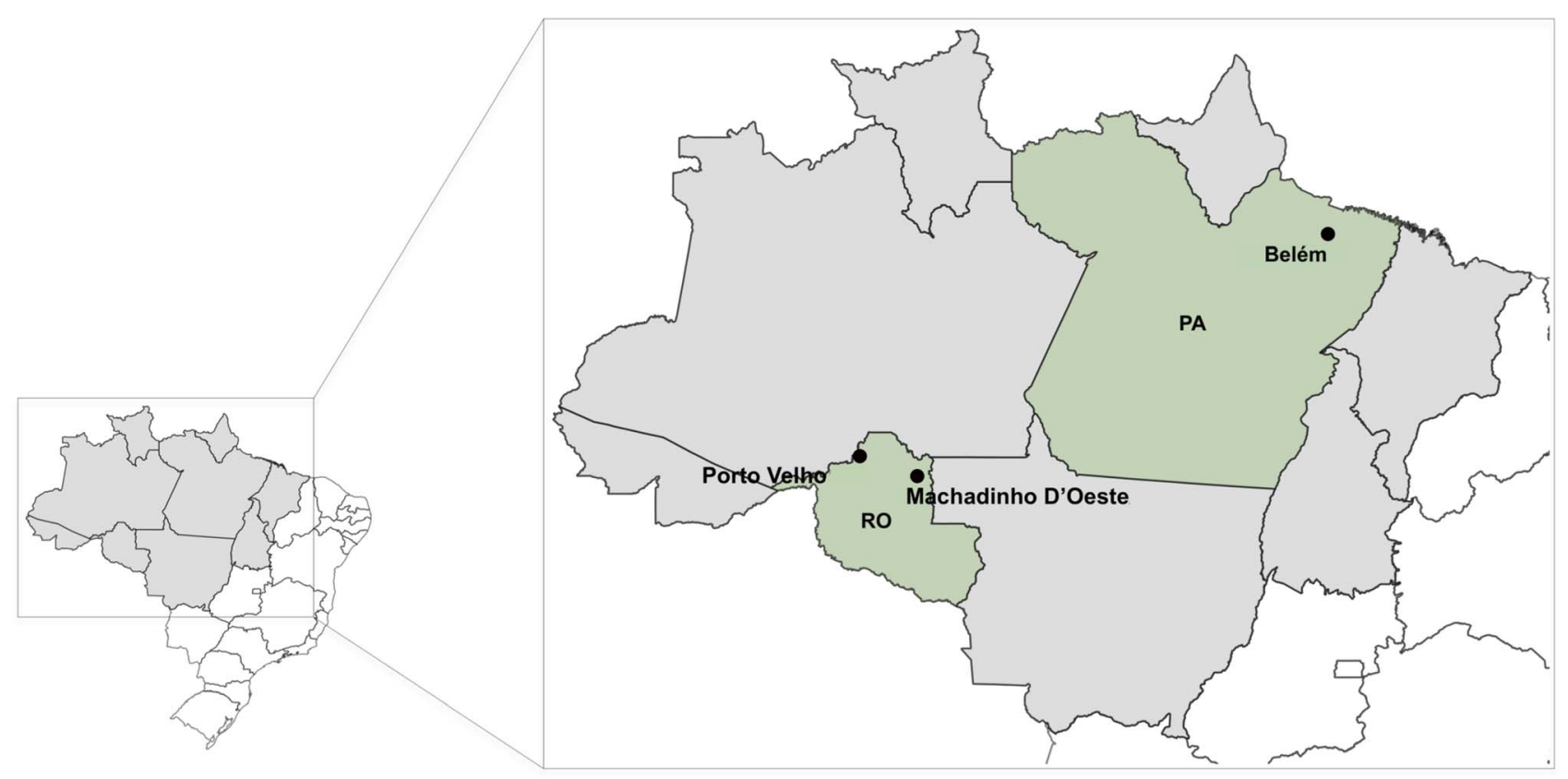

Figure 1. Map of Brazil showing the localities where Leishmania (Viannia) lindenbergi was already detected. The Amazon region is presented in grey, and in green are the two States ( $\mathrm{PA}=$ Pará; $\mathrm{RO}=$ Rondônia) showing the municipalities that presented cases of human cutaneous leishmaniasis caused by L. lindenbergi.

the Rondônia Reference Hospital for Tropical Medicine (CEMETRON). In August 2014, a 32-year-old male patient (Patient 1) presenting cutaneous lesions for approximately 1 year and 8 months that were located on the left arm, was seen at CEMETRON. The patient reported that the infection was acquired in a rural area of the municipality of Machadinho D'Oeste, in the state of Rondônia, where he lives and works in agriculture. This municipality is approximately $400 \mathrm{~km}$ from Rondônia's capital, Porto Velho (Fig. 1).

In December 2015, another patient, a 50-year-old woman (Patient 2), with a cutaneous lesion on the left hand (Fig. 2) was seen at CEMETRON. She reported that the infection may be related to labor activities and was acquired on "Estrada do Índio" (Federal Road 319), a federal highway that connects the cities of Porto Velho and Humaitá, in the Brazilian states of Rondônia and Amazonas, respectively (Fig. 1). This highway has an extension of approximately $900 \mathrm{~km}$, is surrounded by Amazon forest, and is an area with frequent reports of TL.

Clinical data and biological samples were collected within the scope of the research project conducted at CEMETRON, and this study was approved by the Ethics Committee under the protocol CAAE 0020.0.046.000-11. Patients were informed about the research project, agreed voluntarily to participate, and signed consent forms. The two patients did not report any chronic disease and the investigation for HIV coinfection was negative. Parasite isolation was performed by inoculating the lesion border aspirate in biphasic culture medium (NNN + Schneider supplemented). Samples for molecular detection and identification of the parasite were collected using sterile cervical brushes placed in direct contact with the edge of the lesion. PCR was performed targeting $k$ DNA [6] and $h s p 70$ [5] for Leishmania detection.

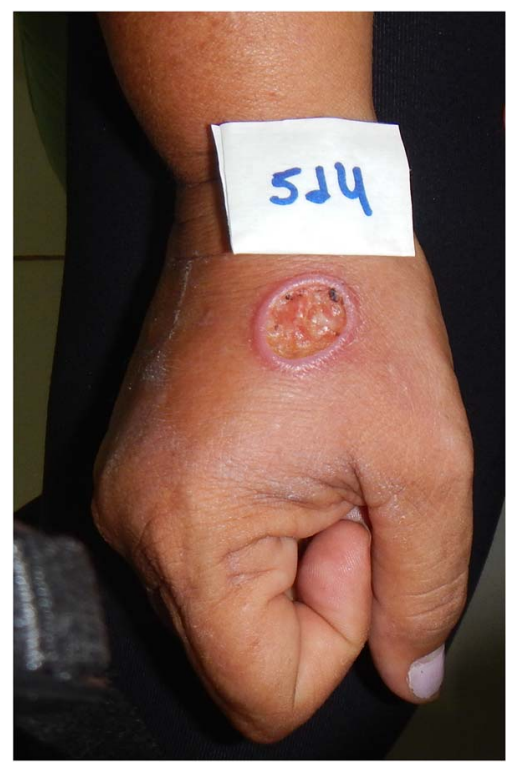

Figure 2. Single cutaneous Leishmaniasis lesion associated with Leishmania lindenbergi observed in a patient (Patient 2) from the State of Rondônia, Brazil. Clinical diagnosis was confirmed by direct and indirect parasitological tests (see Table 1 for details), and Leishmania species identification was achieved by Multilocus Enzyme Electrophoresis (MLEE).

\section{Results and discussion}

Positive results were obtained in all parasitological tests for samples from Patient 2, while for samples from Patient 1, only PCR targeting $k \mathrm{DNA}$ and parasite isolation in culture medium were positive (Table 1). Leishmania parasites were observed for 
Table 1. Description of parasitological tests performed for samples collected in this study.

\begin{tabular}{lccccc}
\hline Sample & Microscopy & PCR $k$ DNA & PCR $h s p 70$ & Parasite isolation & International code \\
\hline Patient 1 & NEG & POS & NEG & POS & MHOM/BR/2014/RO285 \\
Patient 2 & POS & POS & POS & POS & MHOM/BR/2015/RO514 \\
\hline
\end{tabular}

NEG, negative; POS, positive.

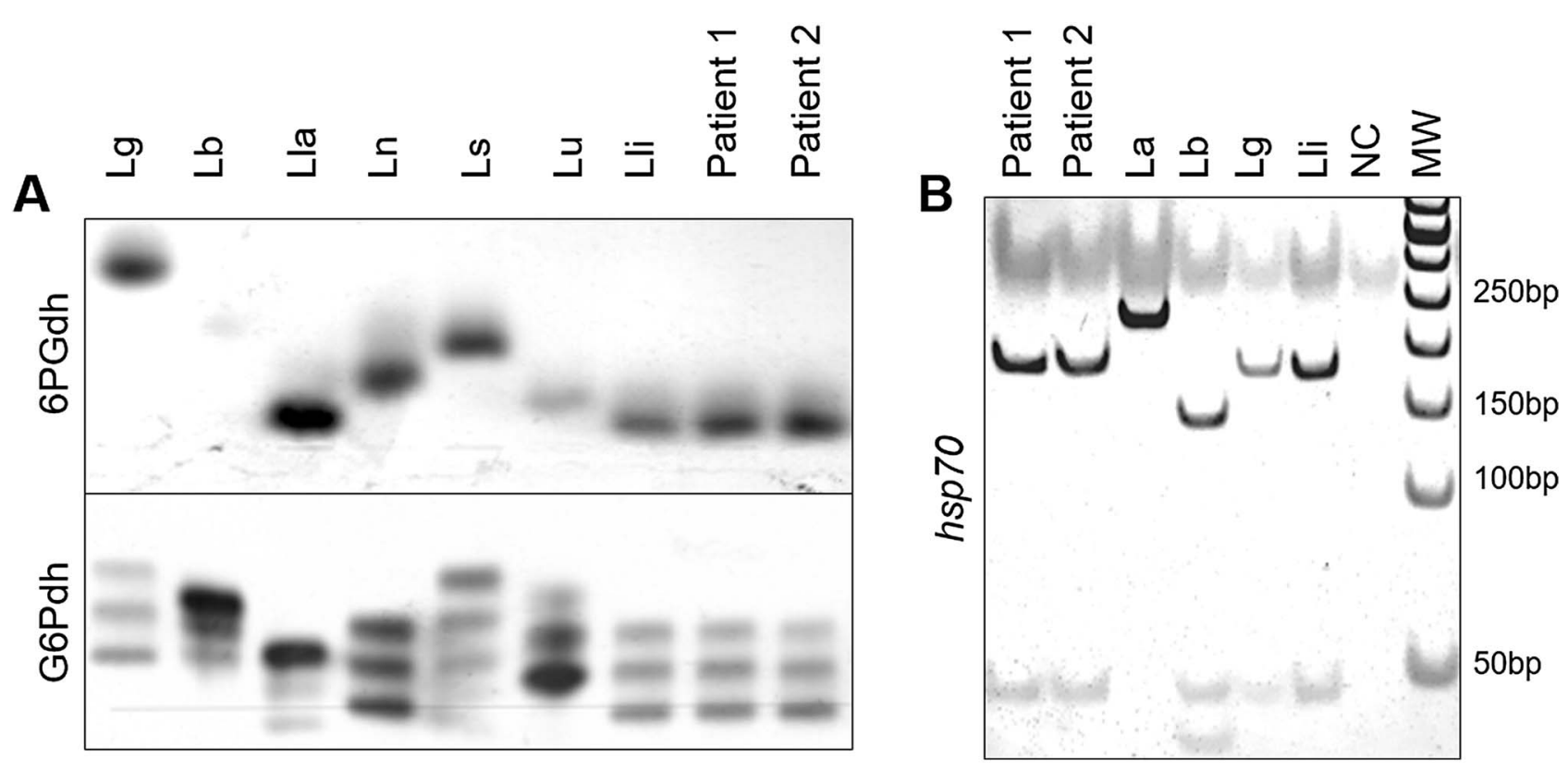

Figure 3. Multilocus Enzyme Electrophoresis (MLEE) and hsp70 PCR-RFLP for Leishmania species identification. (A) Agarose gels stained for activity of 6-phosphogluconate dehydrogenase (6PGD) and glucose-6-phosphate dehydrogenase (G6PD) showing the patterns for Leishmania parasites isolated from Patients 1 and 2. (B) Polyacrylamide gel showing hsp 70 products digested with HaeIII for Leishmania parasites isolated from Patients 1 and 2. In both assays, the profiles obtained for the two samples were compared to reference strains from different Leishmania species. For MLEE, the pattern was compatible with Leishmania (Viannia) lindenbergi. hsp70 PCR-RFLP was not useful for species identification of parasites from the two patients studied, as this marker cannot distinguish between $L$. (V.) lindenbergi and L. (V.) guyanensis. $\mathrm{Lg}=$ L. guyanensis, $\mathrm{Lb}=$ L. braziliensis, Lla $=$ L. lainsoni, $\mathrm{Ln}=$ L. naiffi, $\mathrm{Ls}=$ L. shawi, Lu $=$ L. utingensis, Lli $=$ L. lindenbergi, and $\mathrm{La}=L$. amazonensis $; \mathrm{NC}=$ Negative Control; $\mathrm{MW}=$ Molecular Weight

both isolates in less than 30 days and both cultures reached the amount of parasites needed for cryopreservation approximately 20 days after the first parasite visualization. Both isolates were negative for the presence of the viral endosymbiont Leishmania RNA Virus 1, as determined following protocols described elsewhere [4].

Patient 1 did not return to perform the treatment at CEMETRON hospital and the second patient obtained clinical cure 90 days after the treatment recommended by the Ministry of Health from Brazil (Glucantime ${ }^{\circledR} 15 \mathrm{mg} /$ day for 20 days).

Isolated Leishmania were deposited at the Leishmania Collection of the Oswaldo Cruz Institute (CLIOC) and processed for identification at the species level, employing multilocus isoenzyme electrophoresis (MLEE). Samples were identified as Leishmania (Viannia) lindenbergi (Fig. 3A) and deposited in CLIOC as IOC/L3645 (MHOM/BR/2014/ RO285) and IOC/L3746 (MHOM/BR/2015/RO514).

The positive hsp70 sample (Patient 2) was submitted to RFLP for Leishmania species identification [5]. The profile obtained was compatible with species of the subgenus Viannia, but species identification could not be accomplished. PCR-RFLP of the $h s p 70$ gene was performed employing DNA extracted from Leishmania cultures, the same as used for the isoenzyme assay, and the profiles obtained by HaeIII digestions were compatible with L. lindenbergi and L. guyanensis, as these species presented the same pattern for this marker using this approach (Fig. 3B). A larger portion of the $h s p 70$ gene was amplified and sequenced, following already described protocols [12]. Both sequences were deposited at GenBank under the accession numbers MK792944 (IOC/L3645) and MK792945 (IOC/L3746). The global alignment using BLAST indicated $98.84 \%$ and $99.80 \%$ of identity with the sequence MG029124, a Leishmania lindenbergi sequence publicly available at GenBank, for IOC/L3645 and IOC/L3746, respectively. Another two genes were partially sequenced, isocitrate dehydrogenase (ICD) and mannose phosphate isomerase (MPI) for the two samples, as both regions are useful to distinguish $L$. lindenbergi from the others L. (Viannia) species [1], and the identification of IOC/L3645 and IOC/ L3746 was confirmed as L. lindenbergi (Fig. 4). For ICD, 

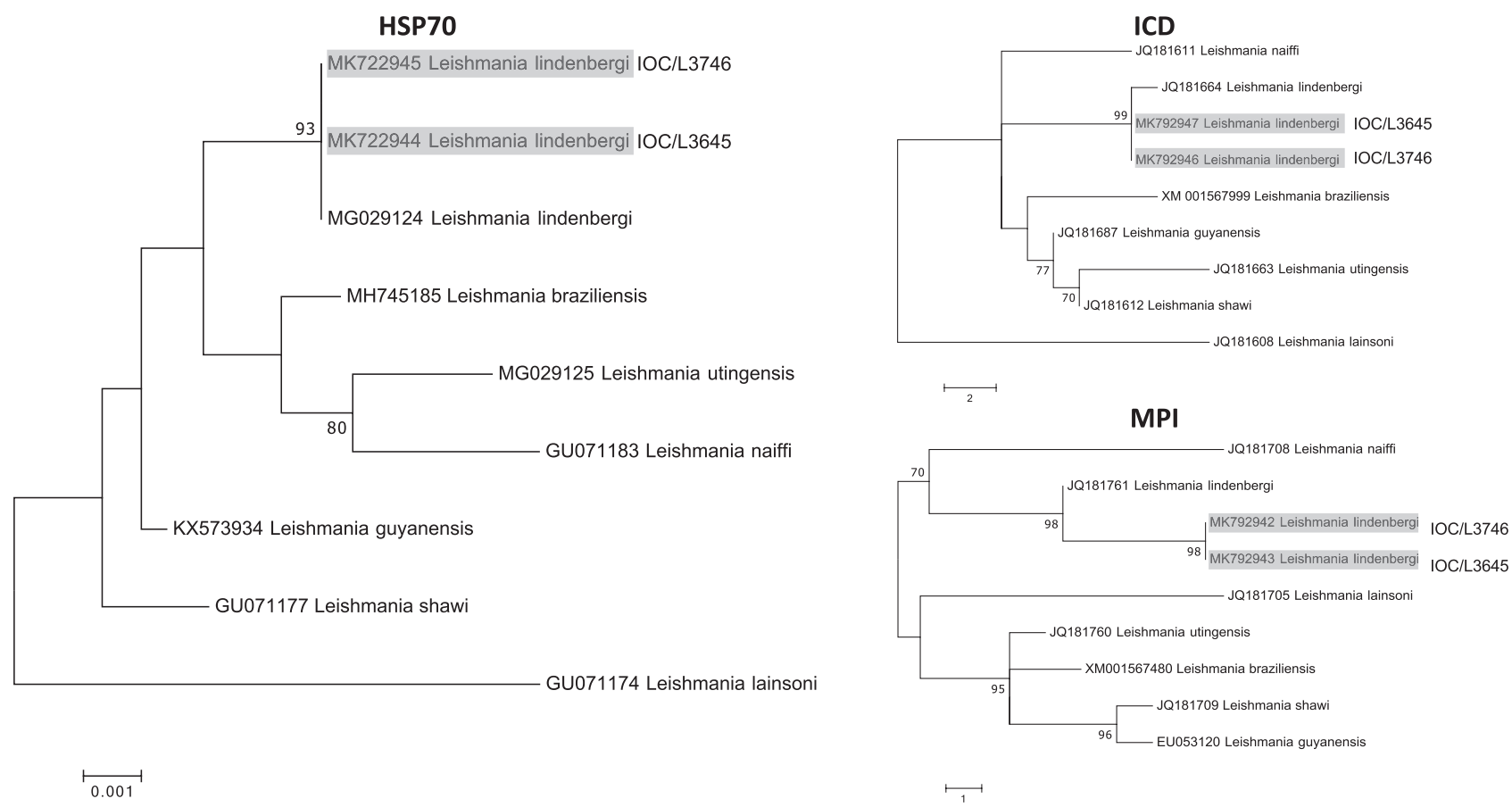

Figure 4. Neighbor-joining trees based on the analysis of partial sequences of $h s p 70$, icd and mpi for L. (Viannia) species, indicating the identity of IOC/3645 and IOC/L3746 with L. lindenbergi. Bootstrap test (1000 replicates) was performed and values above 70\% are shown. The trees are drawn to scale. The evolutionary distances were computed using the number of differences method [8]. GenBank accession numbers for each sequence are presented before the name of the species corresponding to each branch.

IOC/L3645 and IOC/L3746 showed $99.77 \%$ and $98.77 \%$ identity with L. Lindenbergi (GenBank accession number JQ181664), respectively. For MPI, the highest identity observed was also with L. lindenbergi (GenBank accession number JQ181761), $94.36 \%$ and $98.81 \%$ for IOC/L3645 and IOC/L3746, respectively. Cluster analyses were performed to show the similarity of both $L$. lindenbergi strains, from Rondônia State, with Leishmania (Viannia) species circulating in the Amazon region (Fig. 4).

To date, L. lindenbergi has only been observed in human infections within the Brazilian state of Pará (Fig. 1), as presented in a study describing this Leishmania species. Therefore, there is little information describing the biology of this parasite or its transmission cycle [13]. In the description of this species, a similarity was mentioned to others of the same subgenus, such as $L$. naiffi. The molecular protocols used in the present study can distinguish $L$. lindenbergi from $L$. naiffi, but the final identification as $L$. lindenbergi was possible through the analysis of isoenzymes and DNA sequences of $h s p 70$ and housekeeping genes, since PCR-RFLP of $h s p 70$ does not allow discrimination between $L$. lindenbergi and $L$. guyanensis, as already mentioned. The difficulty of properly identifying Leishmania species using the PCR-RFLP hsp70 was recently described, highlighting a limitation of this approach in some circumstances [7]. As presented by Silveira et al. [13], it is possible that L. lindenberg $i$ is associated with more cases of TL if we consider that some methodologies currently employed for Leishmania species identification may not distinguish all species.

Out of seven Leishmania species associated with human TL infections in the North Region of Brazil, only L. lindenbergi had yet to be recorded in the state of Rondônia [3, 4]. In this state, approximately one thousand new cases of TL are registered per year, and the municipalities of Porto Velho and Machadinho d'Oeste comprise $16 \%$ and $4 \%$ of these cases, respectively [2].

The most likely vector of $L$. lindenbergi, $N$. antunesi, is abundant in the rural areas of Porto Velho, representing $25 \%$ of collected sandflies [11]. Natural infection by Leishmania spp. were already reported for this species in a study examining phlebotomines collected inside caves from different areas of Rondônia [10].

The diversity of sand fly vectors and Leishmania species currently described in the Amazon Region hinders efforts to characterize TL. The impossibility of distinguishing between L. lindenbergi and L. guyanensis when using hsp70 PCRRFLP, currently one of the most widespread molecular markers for Leishmania identification, must be taken into account. This issue affects the reports concerning species distribution in endemic areas, which is an important aspect for understanding the epidemiology of TL and is even more relevant in areas with various etiological agents.

Few studies have addressed the taxonomic status of L. lindenbergi, and a genetic relationship of this species with L. naiffi, a genetically polymorphic parasite, has already been demonstrated [1]. Performing further studies to investigate the origin and genetic relationships of poorly studied species, such as $L$. lindenbergi, is crucial. For now, we can state that parasites with the genetic profile of $L$. lindenbergi could be widespread in distinct regions of the Brazilian Amazon, but further investigations are needed to confirm the distribution and frequency of this species. 
Acknowledgements. We declare that all patients agreed to be included in this study. The authors declare no conflict of interest during the development of the present study. This study was supported by fellowships and grants from the National Council for Scientific and Technological Development (CNPq), Fundação Rondônia de Amparo ao Desenvolvimento das Ações Científicas e Tecnológicas e à Pesquisa do Estado de Rondônia (FAPERO), Fundação Carlos Chagas de Amparo a Pesquisa do Estado do Rio de Janeiro (FAPERJ), and the National Institute of Epidemiology of the Brazilian Western Amazon (EpiAmO).

\section{References}

1. Boité MC, Mauricio IL, Miles MA, Cupolillo E. 2012. New Insights on taxonomy, phylogeny and population genetics of Leishmania (Viannia) parasites based on multilocus sequence analysis. PLoS Neglected Tropical Diseases, 6(11), e1888.

2. Brasil. Ministério da Saúde. 2018. Leishmaniose Tegumentar Americana - Casos confirmados Notificados no Sistema de Informação de Agravos de Notificação - Sinan Net: 2018.

3. Cantanhêde LM, Fernandes FG, Ferreira GEM, Porrozzi R, Ferreira RDGM, Cupolillo E. 2018. New insights into the genetic diversity of Leishmania RNA Virus 1 and its speciesspecific relationship with Leishmania parasites. PLoS One, 13 (6), e0198727.

4. Cantanhêde LM, Silva Júnior CF, Ito MM, Felipin KP, Nicolete R, Salcedo JMV, Porrozzi R, Cupolillo E, Ferreira RGM. 2015. Further evidence of an association between the presence of Leishmania RNA Virus 1 and the mucosal manifestations in tegumentary leishmaniasis patients. PLoS Neglected Tropical Diseases, 9(9), e0004079.

5. da Graça GC, Volpini AC, Romero GAS, Oliveira Neto MPD, Hueb M, Porrozzi R, Boité MC, Cupolillo E. 2012. Development and validation of PCR-based assays for diagnosis of American cutaneous leishmaniasis and identification of the parasite species. Memórias do Instituto Oswaldo Cruz, 107, 664-674.
6. Degrave W, Fernandes O, Campbell D, Bozza M, Lopes U. 1994. Use of molecular probes and PCR for detection and typing of Leishmania - A mini-review. Memórias do Instituto Oswaldo Cruz, 89, 463-469.

7. Espada CR, Ortiz PA, Shaw JJ, Barral AMP, Costa JML, Uliana SRB, Coelho AC. 2018. Identification of Leishmania (Viannia) species and clinical isolates of Leishmania (Leishmania) amazonensis from Brazil using PCR-RFLP of the heat-shock protein 70 gene reveals some unexpected observations. Diagnostic Microbiology and Infectious Disease, 91(4), 312-318.

8. Kumar S, Stecher G, Tamura K. 2016. MEGA7: Molecular evolutionary genetics analysis version 7.0 for bigger datasets. Molecular Biology and Evolution, 33, 1870-1874.

9. Lainson R. 2010. The neotropical Leishmania species: A brief historical review of their discovery, ecology and taxonomy. Revista Pan-Amazônica de Saúde, 1(2), 13-32.

10. Ogawa GM, Pereira Júnior AM, Resadore F, Ferreira RDGM, Medeiros JF, Camargo LMA. 2016. Sandfly fauna (Diptera: Psychodidae) from caves in the state of Rondônia, Brazil. Revista Brasileira de Parasitologia Veterinária, 25(1), 61-68.

11. Resadore F, Pereira Júnior AM, Carvalho LPC, dos Santos APDA, Teles CBG, Medeiros JF. 2017. Phlebotomine sand fly composition (Diptera: Psychodidae) and putative vectors of American cutaneous leishmaniasis in Porto Velho municipality, Western Amazon, Brazil. Journal of Medical Entomology, 54(3), 798-803.

12. Silva LA, Souza CS, Graça GC, Porrozzi R, Cupolillo E. 2010. Sequence analysis and PCR-RFLP profiling of the hsp70 gene as a valuable tool for identifying Leishmania species associated with human leishmaniasis in Brazil. Infection, Genetics and Evolution, 10, 77-83.

13. Silveira FT, Ishikawa EAY, De Souza AAA, Lainson R. 2002. An outbreak of cutaneous leishmaniasis among soldiers in Belém, Pará State, Brazil, caused by Leishmania (Viannia) lindenbergi. Parasite, 9(1), 43-50.

14. Silveira FT, Lainson R, Corbett CE. 2004. Clinical and immunopathological spectrum of American cutaneous leishmaniasis with special reference to the disease in Amazonian Brazil - a review. Memórias do Instituto Oswaldo Cruz, 99, 239-251.

Cite this article as: Cantanhêde LM, Mattos CB, de Souza Ronconi C, Filgueira CB, da Silva Júnior CF, Limeira C, de Jesus Silva HP, Ferreira GEM, Porrozzi R, de Godoi Mattos Ferreira R \& Cupolillo E. 2019. First report of Leishmania (Viannia) lindenbergi causing tegumentary leishmaniasis in the Brazilian western Amazon region. Parasite 26, 30.

\section{PARASTTE}

An international open-access, peer-reviewed, online journal publishing high quality papers on all aspects of human and animal parasitology

Reviews, articles and short notes may be submitted. Fields include, but are not limited to: general, medical and veterinary parasitology; morphology, including ultrastructure; parasite systematics, including entomology, acarology, helminthology and protistology, and molecular analyses; molecular biology and biochemistry; immunology of parasitic diseases; host-parasite relationships; ecology and life history of parasites; epidemiology; therapeutics; new diagnostic tools.

All papers in Parasite are published in English. Manuscripts should have a broad interest and must not have been published or submitted elsewhere. No limit is imposed on the length of manuscripts.

Parasite (open-access) continues Parasite (print and online editions, 1994-2012) and Annales de Parasitologie Humaine et Comparée (1923-1993) and is the official journal of the Société Française de Parasitologie. 